\title{
Efeito de micronutrientes sobre o crescimento de mudas de mogno (Swietenia macrophilla King) em Latossolo amarelo
}

\author{
Wellington Gomes da SILVA¹, Carlos Alberto Franco TUCCI, Francisco Adilson dos Santos HARA, \\ Renata Alexandra Cabrita dos SANTOS
}

\section{RESUMO}

O plantio de espécies florestais nativas é uma atividade que além de repor os recursos florestais também pode atenuar os impactos ambientais decorrentes do extrativismo. Entretanto, seu sucesso depende, entre outros fatores, do conhecimento a cerca das necessidades nutricionais da espécie a ser utilizada. Com o objetivo de obter informaçôes das necessidades de mudas de mogno (Swietenia macrophilla King) por micronutrientes, foi realizado um experimento em casa de vegetação. Foi utilizado como substrato um Latossolo Amarelo de baixa fertilidade, coletado da camada de 20-40 cm de profundidade, localizado no setor Sul do Campus da Universidade Federal do Amazonas (UFAM). Foram testados sete tratamentos e quatro repetições dispostos em delineamento experimental de blocos ao acaso, sob a técnica do elemento faltante. Os tratamentos foram: Completo (Calagem + N, P, K, Ca, Mg, S, B, Cu, Fe, Mn, Zn e Mo), Testemunha (Calagem + N, P, K, Ca, Mg, S e Mo) e a omissão de um micronutriente catiônico por vez (-B, $-\mathrm{Cu},-\mathrm{Fe},-\mathrm{Mn}$ e - $\mathrm{Zn})$. Após quatro meses foram avaliadas as seguintes características: altura da parte aérea, diâmetro do colo, produção de matéria seca da parte aérea e das raízes e conteúdo de nutrientes na matéria seca da parte aérea. Os resultados obtidos nesta pesquisa permitiram concluir que as mudas de mogno tiveram seu crescimento comprometido pela baixa disponibilidade de cobre no substrato, sendo necessária sua aplicação para que as plantas tenham um desenvolvimento normal, compatível com o crescimento da espécie, quando as condiçóes de substrato são adequadas.

\section{PALAVRAS-CHAVE}

Nutrição de plantas, Cobre, Swietenia macrophilla.

\section{Effect of micronutrients in oxisoil in the growth of seedling mahogany (Swietenia Macrophilla King)}

\begin{abstract}
The planting of native forest species is an activity which in addition to replacing forest resources can also lessen the environmental impacts resulting from their extraction. However, its success depends, among other things, on the knowledge regarding the nutritional needs of the species to be utilized. An experiment was carried out in a greenhouse aiming to acquire information on the mahogany (Swietenia macrophilla) seedling micronutrient requirements. A low fertility Yellow Latosol, collected from a $20-40 \mathrm{~cm}$ deeplayer, located at the University Federal of Amazonas (UFAM) Campus southern sector, was used as substract. We tested seven treatments an four replicated arranged in a random block experimental design, under the lacking element technique. Treatments were as follows (Liming + N, P, K, Ca, Mg, S, B, Cu, Fe, Mn, Zn and Mo), and the omission of a catiônico micronutrient at a time (-B, $-\mathrm{Cu},-\mathrm{Fe},-\mathrm{Mn}$ and $-\mathrm{Zn})$. The following features were assessed after four months: aerial part height, diameter, and aerial part and root dry matter yield. The resulted obtained in this research allowed to conclude that the mahogany seedlings had their growth compromised by the low availability of cooper in the substract, thus its application is necessary so that the plants would a normal growing, compatible with the their usual growth, when the substract conditions were appriate.
\end{abstract}

KEYWORDS

Plant nutrition, Cupper, Swietenia macrophila.

${ }^{1}$ Engenheiro Agrônomo, Msc. Departamento de Engenharia Agrícola e Solos/UFAM. Universidade Federal do Amazonas, Instituto de Ciências Biológicas. Av. Gen. Rodrigo Otávio J. Ramos, 3000 - Coroado. CEP. 69077-000 - Manaus, AM - Brasil e-mail: wellington1111@hotmail.com 


\section{INTRODUÇÃO}

O mogno (Swietenia macrophylla King, Meliaceae) conhecido vulgarmente como aguano, caraputanga, cedro-i, mogno-brasileiro, é uma espécie semidecídua ou decídua, podendo atingir até $30 \mathrm{~m}$ de altura e $80 \mathrm{~cm}$ de DAP. Ocorre em toda região amazônica, sendo mais freqüente na região sul do Pará. É característica do ecossistema de terra firme e classificada como espécie clímax (Lorenzi, 1998).

Segundo Lamprecht (1990) o crescimento do mogno, em condições favoráveis, é muito rápido. A viabilidade de sementes na época da dispersão é geralmente superior a 90\%. As sementes não têm mecanismo de dormência a longo prazo, mas podem sobreviver quatro a seis meses no campo durante o período seco (Gullison et al., 1996) e até dez meses em condições de seca e frio.

Devido ao valor econômico de sua madeira no mercado, essa espécie tem sido explorada em larga escala. Sua exploração tem ocorrido de forma predatória, pois não se tem o cuidado de reposição da espécie e ainda há destruição das plantas, ocasionado pela queda e arraste das arvores, após o corte para retirada das toras (Veríssimo et al.,1995).

O plantio de espécies florestais nativas é uma atividade que pode repor e manter não só os recursos florestais, como também conservar o equilíbrio econômico, social e ambiental (Barros, 2001). Entretanto, o sucesso de um florestamento ou reflorestamento depende além de outros fatores, do conhecimento a cerca das necessidades nutricionais da espécie a ser utilizada (Sanginga et al., 1991).

Informações sobre as necessidades nutricionais das plantas são importantes para conhecer sua demanda por nutrientes e determinar a quantidade correta de fertilizantes a ser empregada, quando o solo não for capaz de suprir essa necessidade (Braga, 1983).

A avaliação das exigências nutricionais das plantas pode envolver aspectos quantitativos ou qualitativos (Malavolta, 1980). Como passo inicial, a avaliação em caráter qualitativo constitui-se numa ferramenta simples e eficiente, fornecendo subsídios para estudos posteriores de cunho quantitativo utilizando-se comumente a técnica do elemento faltante (Sanches, 1981; Raij, 1991).

A diagnose por subtração, ou técnica do elemento faltante pode fornecer informaçôes qualitativas a cerca dos nutrientes que podem limitar o desenvolvimento das plantas (Braga, 1983; Lopes \& Carvalho, 1987).

Com o objetivo de verificar os efeitos da omissão de nutrientes na nutrição mineral do pinheiro (Araucária angustifólia), Simóes \& Couto (1973) concluíram que a falta de $\mathrm{N}, \mathrm{P}, \mathrm{K}, \mathrm{Ca}$ e $\mathrm{Mg}$ comprometeram o desenvolvimento das plantas.

As exigências nutricionais de quatro espécies florestais foram avaliadas por Braga et al. (1995) em um solo de baixa fertilidade. Esses autores observaram que essas espécies apresentaram comportamento diferenciado. A quaresmeira (Tiboucha granulosa) apresentou o maior requerimento nutricional, tendo seu desenvolvimento limitado pela omissão de todos os macro e micronutrientes. A pereira (Platycyamus regnelli), pelo N, P, K, Ca, e S. A peroba rosa (Aspidosperma polineurom) pelo $\mathrm{P}, \mathrm{K}$ e S e a Acácia mangium pelo N, P e S.

Renó et al. (1997), utilizando a técnica do nutriente faltante concluíram que para o crescimento em altura do Cedro (Cedrella fissiliis), do jacaré (Piptadenia gonoacantha), do pau-ferro (Calsapinea férrea) e da canafístula (Senna multijuga), o P, S e N foram limitantes e o K não se mostrou limitante a nenhuma delas, evidenciando assim baixo requerimento para esse nutriente. Entretanto, essas espécies apresentaram comportamento diferenciado para a omissão de micronutrientes. Os micronutrientes mostaram-se limitantes ao crescimento do pau-ferro e da canafístula enquanto o cedro e a canafístula mostraram-se indiferentes.

Os requerimentos nutricionais da aroeira do sertão (Myracrodruon urundeuva) foram estudados por Mendonça et al. (1999) utilizando a técnica da diagnose por subtração. Esses autores concluíram que a omissão de N, P, K, Ca e Mg foram limitantes ao desenvolvimento das plantas, enquanto a omissão de $\mathrm{Cu}, \mathrm{Fe}, \mathrm{Mn}$ e $\mathrm{Zn}$ não afetou o crescimento das mudas até o quarto mês após a repicagem.

$\mathrm{Na}$ literatura, poucas são as informaçōes sobre as necessidades nutricionais do mogno (Swietenia macrophylla), podendo citar as pesquisas desenvolvidas por Pinto et al. (2000), Barros (2001), Silva (2004) e Santos (2006), direcionadas para recomendação de calagem, nitrogênio, fósforo e potássio na fase de produção de mudas, não sendo encontrado nenhum trabalho sobre a exigência dessa espécie por micronutrientes.

O objetivo dessa pesquisa foi avaliar a disponibilidade de micronutrientes catiônicos em um Latossolo Amarelo para o desenvolvimento das plantas de mogno na fase de muda.

\section{MATERIAL E MÉTODOS}

O experimento foi conduzido em casa de vegetação, na Faculdade de Ciências Agrárias da Universidade Federal do Amazonas (UFAM), no período de julho a outubro de 2004 , utilizando-se como substrato um Latossolo Amarelo de baixa fertilidade natural, retirado da camada subsuperficial de 20 a $40 \mathrm{~cm}$ de profundidade, no município de Manaus (Am), situada no Setor Sul do Campus da Universidade Federal do Amazonas, cujas coordenadas geográficas, em UTM, são 21 M 016894 e 9657166. 
O solo foi seco ao ar, destorroado em peneira com abertura de 4,0 $\mathrm{mm}$ de malha, pesado, adicionado calcário, acondicionado em sacos plásticos e incubado por 30 dias (para reação do corretivo com o solo). Foram tomadas amostras para determinação da textura, $\mathrm{pH}\left(\mathrm{H}_{2} \mathrm{O}\right)$, matéria orgânica, $\mathrm{Ca}^{2+}, \mathrm{Mg}^{2+}, \mathrm{Al}^{3+}, \mathrm{H}+\mathrm{Al}, \mathrm{Cu}, \mathrm{Fe}, \mathrm{Mn}$ e $\mathrm{Zn}$, antes da adição de calcário e dez dias após a aplicação de fertilizantes, (Tabela 1). Essas análises foram realizadas no Laboratório de Solos da EMBRAPA-CPAA (Manaus), segundo a metodologia da EMBRAPA (1999).

Tabela 1 - Características do Latossolo Amarelo utilizado como substrato para 0 crescimento de mudas de mogno.

\begin{tabular}{|c|c|c|}
\hline Parâmetros & Substrato antes da correção & Substrato corrigido \\
\hline $\mathrm{pH}\left(\mathrm{H}_{2} \mathrm{O}\right)$ & 3,55 & 4,16 \\
\hline$C\left(g \cdot \mathrm{kg}^{-1}\right)$ & 13,4 & 13,22 \\
\hline M.O $\left(\mathrm{g}^{\mathrm{kg}} \mathrm{g}^{-1}\right)$ & 23,1 & 22,74 \\
\hline$P\left(m g . d m^{-3}\right)$ & 2,00 & 168,00 \\
\hline $\mathrm{K}\left(\mathrm{mg} \cdot \mathrm{dm}^{-3}\right)$ & 11,0 & 15,00 \\
\hline $\mathrm{Ca}\left(\mathrm{cmol}_{\mathrm{c}} \cdot \mathrm{dm}^{-3}\right)$ & 0,05 & 1,35 \\
\hline $\mathrm{Mg}\left(\mathrm{cmol}_{\mathrm{c}} \cdot \mathrm{dm}^{-3}\right)$ & 0,04 & 0,36 \\
\hline $\mathrm{Al}\left(\mathrm{cmol}_{\mathrm{c}} \cdot \mathrm{dm}^{-3}\right)$ & 1,40 & 0,37 \\
\hline $\mathrm{H}+\mathrm{Al}\left(\mathrm{cmol}_{\mathrm{c}} \cdot \mathrm{dm}^{-3}\right)$ & 6,64 & 5,87 \\
\hline $\mathrm{Cu}\left(\mathrm{mg}_{\mathrm{dm}}^{-3}\right)$ & 0,08 & 0,90 \\
\hline $\mathrm{Fe}\left(\mathrm{mg} \cdot \mathrm{dm}^{-3}\right)$ & 85,0 & 103 \\
\hline $\operatorname{Mn}\left(\mathrm{mg}_{\mathrm{dm}} \mathrm{dm}^{-3}\right)$ & 0,62 & 3,56 \\
\hline $\mathrm{Zn}\left(\mathrm{mg} \cdot \mathrm{dm}^{-3}\right)$ & 0,62 & 4,9 \\
\hline Areia $\left(\mathrm{g} \cdot \mathrm{kg}^{-1}\right)$ & 157,1 & - \\
\hline Silte $\left(g \cdot k g^{-1}\right)$ & 17,5 & - \\
\hline Argila $\left(g \cdot \mathrm{kg}^{-1}\right)$ & 802,4 & - \\
\hline
\end{tabular}

Foram utilizadas sementes ( $70 \%$ de germinação e 99,5\% de pureza) provenientes do município de Paragominas - PA, obtidas junto a Associação das Indústrias Exportadoras de Madeira do Estado do Pará - AIMEX. A semeadura, feita em copos descartáveis com areia lavada com água mineral. Após o aparecimento de dois pares de folhas as plântulas foram repicadas para vasos com capacidade para $12 \mathrm{dm}^{3}$. A umidade do solo foi mantida com $80 \%$ da capacidade de campo e aferida diariamente através de pesagem.

Foram avaliados sete tratamentos e quatro repetiçôes, perfazendo um total de 28 unidades experimentais analisadas em delineamento experimental de blocos ao acaso, sendo cada unidade experimental formada por duas plantas. Os tratamentos foram: 1) Completo (Calagem + N, P, K, Ca, Mg, $\mathrm{S}, \mathrm{B}, \mathrm{Cu}, \mathrm{Fe}, \mathrm{Mn}, \mathrm{Zn}$ e Mo), 2) Testemunha (Calagem + N, $\mathrm{P}, \mathrm{K}, \mathrm{Ca}, \mathrm{Mg}, \mathrm{S}$ e Mo), 3) Completo menos B, 4) Completo menos $\mathrm{Cu}$, 5) Completo menos $\mathrm{Fe}$ ), 6) Completo menos $\mathrm{Mn}$ e 7) Completo menos Zn.

Para correção do substrato aplicou-se a quantidade equivalente a 2 t.ha- ${ }^{-1}$ de corretivo, utilizando-se uma mistura de $\mathrm{CaCO}_{3}$ e $\mathrm{MgCO}_{3}$ p.a., na proporção de 4:1 equivalentes, aplicada 45 dias antes da repicagem, mantendo-se a umidade do substrato constante, com água deionizada, durante o período de incubação. Após a incubação foi realizada a adubação do substrato, realizada de acordo com Novais et al. (1991); a concentração dos nutrientes utilizados para a adubação básica, expressa em mg. $\mathrm{kg}^{-1}$ de substrato foi: $100 \mathrm{de}$ N 300 de P, 150 de K, 40 de S, 1 de B, 1,5 de Cu, 5 de Fe, 5 de $\mathrm{Mn}, 5$ de $\mathrm{Zn}$ e $0,15 \mathrm{Mo}$. O N e o $\mathrm{K}$ foram parcelados (Malavolta, 1980) em 3 aplicações, sendo uma no preparo do substrato e as duas restantes em cobertura, 15 e 30 dias após a repicagem. Exceto para o P (superfosfato simples) e $\mathrm{K}$ (cloreto de potássio), a fonte de nutrientes utilizada foram reagentes p.a.: $\mathrm{N}\left(\mathrm{NH}_{2} \mathrm{CoNH}_{2}\right), \mathrm{B}\left(\mathrm{H}_{3} \mathrm{BO}_{3}\right), \mathrm{Cu}$ $\left(\mathrm{CuCl}_{2} \cdot 2 \mathrm{H}_{2} \mathrm{O}\right), \mathrm{Fe}\left(\mathrm{FeSO}_{4} \cdot 7 \mathrm{H}_{2} \mathrm{O}\right), \mathrm{Mn}\left(\mathrm{MnCl}_{2} \cdot 4 \mathrm{H}_{2} \mathrm{O}\right), \mathrm{Mo}$ $\left[\left(\mathrm{NH}_{4}\right)_{6} \mathrm{Mo}_{7} \mathrm{O}_{24} \cdot 4 \mathrm{H}_{2} \mathrm{O}\right)$ e $\mathrm{Zn}\left(\mathrm{ZnCl}_{2}\right)$.

Aos 120 dias após a repicagem foram avaliados o diâmetro do colo, a altura da parte aérea e a produção da matéria seca da parte aérea, das raízes e total. $\mathrm{O}$ diâmetro foi tomado no coleto; e a altura, do solo até o a inserção da última folha. Após tomadas as medidas, as mudas foram cortadas rente ao solo. O sistema radicular foi separado do solo com jatos de água mineral. Ambas, raízes e parte aérea foram secas em estufa com circulação forçada de ar a $70^{\circ} \mathrm{C}$, até obtenção de massa constante, para determinação da massa da matéria seca e pelo somatório da massa da raiz e da parte aérea, o massa seca total. A relação raiz: parte aérea foi calculada dividindo-se a massa seca da raiz pela massa seca da parte aérea.

O efeito relativo foi calculado pelo método de percentagem de suficiência ou produção relativa (Raij, 1991), adaptado para a determinação do "crescimento relativo" (CR), calculado da seguinte forma: o crescimento relativo é igual ao valor da massa seca total do tratamento com o nutriente omitido, multiplicado por cem e dividido pelo valor da massa seca do tratamento completo, pois para o tratamento completo considera-se que seu crescimento relativo foi de $100 \%$.

$\mathrm{O}$ efeito dos tratamentos foi submetido à análise de variância e o teste de médias utilizado foi o de Tukey $(\mathrm{p}<0,01)$, conforme descrito por Gomes (1991). Para a realização dos cálculos estatísticos foi utilizado o Programa computacional Sisvar (Ferreira, 2000).

\section{RESULTADOS E DISCUSSÃO}

Verifica-se que o tratamento com omissão de cobre e a Testemunha restringiram o crescimento em altura, diâmetro do colo, produção de matéria seca da parte aérea, do sistema radicular e matéria seca total das mudas de mogno, enquanto os demais não apresentaram limitações, quando comparados ao completo (Tabela 2). Além de afetar as características de crescimento a omissão de cobre e a testemunha ainda comprometeram o aspecto visual das plantas, com sintomas 
visuais semelhantes aos descrito por Malavolta et al. (1989). Esses resultados sugerem que a omissão de cobre foi responsável pela redução no crescimento das plantas, pois a omissão de $\mathrm{B}, \mathrm{Fe}, \mathrm{Mn}$ e $\mathrm{Zn}$ isolados não afetaram as características avaliadas, visto que seus resultados foram iguais ao tratamento completo (Tabela 2). Além disso, a Testemunha, que não teve cobre adicionado ao substrato apresentou desenvolvimento significativamente igual ao tratamento com omissão desse nutriente, o que reforça mais essa afirmativa.

Tabela 2 - Características de crescimento das mudas de mogno.

\begin{tabular}{|c|c|c|c|c|c|c|c|}
\hline \multirow{2}{*}{ Tratamento } & Altura & Diâmetro & MSPA & MSSR & \multirow{2}{*}{ MST } & \multirow{2}{*}{$\mathrm{R} / \mathrm{PA}$} & \multirow{2}{*}{$\begin{array}{l}\text { CR } \\
(\%)\end{array}$} \\
\hline & $(\mathrm{cm})$ & $(\mathrm{mm})$ & ----- g/u. & e ----- & & & \\
\hline Completo & $27,14 \mathrm{a}$ & $4,88 \mathrm{~b}$ & $13,70 a b$ & $4,28 \mathrm{a}$ & $17,98 \mathrm{ab}$ & $0,31 \mathrm{a}$ & 100 \\
\hline Testemunha & $15,59 b$ & $3,14 \mathrm{a}$ & $3,25 \mathrm{c}$ & $1,00 \mathrm{~b}$ & $4,25 \mathrm{c}$ & $0,31 \mathrm{a}$ & 23,6 \\
\hline$-B$ & $27,81 \mathrm{a}$ & $5,24 \mathrm{~b}$ & $15,35 \mathrm{a}$ & $4,35 \mathrm{a}$ & $19,70 \mathrm{a}$ & $0,29 a$ & 109,6 \\
\hline - $\mathrm{Cu}$ & $16,58 \mathrm{~b}$ & $2,92 \mathrm{a}$ & $2,33 \mathrm{c}$ & $0,73 \mathrm{~b}$ & $3,06 \mathrm{c}$ & $0,31 \mathrm{a}$ & 17,0 \\
\hline - Fé & $27,60 \mathrm{a}$ & $4,98 \mathrm{~b}$ & $14,95 \mathrm{a}$ & $4,68 \mathrm{a}$ & $19,63 \mathrm{a}$ & $0,31 \mathrm{a}$ & 109,2 \\
\hline$-M n$ & $22,19 \mathrm{a}$ & $4,78 \mathrm{~b}$ & $10,59 \mathrm{~b}$ & $3,45 \mathrm{a}$ & $14,04 \mathrm{~b}$ & $0,31 \mathrm{a}$ & 78,1 \\
\hline$-Z n$ & $24,56 \mathrm{a}$ & $4,79 \mathrm{~b}$ & $12,93 a b$ & $3,60 \mathrm{a}$ & $16,53 \mathrm{ab}$ & $0,28 a$ & 91,9 \\
\hline $\mathrm{CV}$ & 10,33 & 11,25 & 15,28 & 27,46 & 17,17 & 23,47 & - \\
\hline
\end{tabular}

Médias seguidas pela mesma letra na coluna não diferem significativamente, a 1\% de probabilidade, pelo teste de Tukey. 0 sinal negativo indica a omissão do nutriente. * Matéria seca do sistema radicular (MSSR), matéria seca da parte aérea (MSPA) matéria seca total (MST), relação raiz/parte aérea (R/PA) e crescimento relativo (CR), referente à matéria seca total.

$\mathrm{Na}$ Tabela 2 observa-se que as mudas de mogno submetidas a omissão de cobre tiveram um crescimento em altura e diâmetro e produção de matéria seca inferior, comparado ao tratamento completo, tomado como referência. Transformando os valores em crescimento relativo, observa-se uma redução de 39\% na altura; 40\% no diâmetro; $83 \%$ na matéria seca da parte aérea e sistema radicular. Essas reduções nas características de crescimento, podem ser explicadas pelo baixo teor de $\mathrm{Cu}^{2+}$ no solo estudado, de $0,08 \mathrm{mg} \cdot \mathrm{dm}^{-3}$, sendo o teor adequado de 1,3 a $1,8 \mathrm{mg} \cdot \mathrm{dm}^{-3}$ para os solos de um modo geral, segundo Alvarez et al., (1999). Resultados semelhantes foram obtidos por Viegas et al. (2004) ao avaliarem as limitaçôes nutricionais das plantas de açaí (Euterpe oleracea Mart.). Esses autores observaram que a omissão de $\mathrm{Cu}$ foi limitante a produção de matéria seca das folhas, caule, parte aérea e total de matéria seca das folhas, caule, parte aérea e

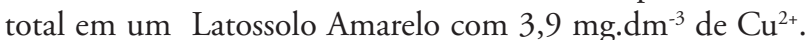
Braga et al. (1995), em experimento com nutriente faltante, em solo de baixa fertilidade, também relataram que o $\mathrm{Cu}$ foi limitante ao desenvolvimento da quaresmeira (Tibouchina granulosa).

As mudas de mogno cultivadas sob as omissóes de boro e ferro apresentaram uma tendência de maior crescimento em altura, diâmetro e produção de matéria seca (Tabela 2) em relação ao tratamento completo. Produçōes gerais entre ausentes e completos indicam que o teor disponível desses nutrientes no solo foram suficiente para atender as exigências nutricionais das plantas. Esse resultado concorda em parte com os resultados obtidos por Duboc et al. (1996), nos quais o diâmetro, a produção de matéria seca da parte aérea e a matéria seca do sistema radicular das mudas de jatobá (Hymenaea courbaril) também apresentaram tendência de crescimento na omissão de boro, quando comparado ao tratamento completo.

As omissōes de manganês e de zinco não afetaram as características de crescimento das plantas, pois as médias de altura, diâmetro e produção de matéria seca das mudas não diferiram do tratamento completo (Tabela 2). Pode-se explicar tal fato, admitindo-se que a concentração de boro e ferro acumulado na semente e/ou os teores disponíveis no solo tenha sido mobilizado para todos os órgãos da planta satisfazendo suas necessidades.

Pesquisas conduzidas por Camargo et al. (2002), em solução nutritiva, com castanheira-do-brasil, mostraram que a omissão de zinco não foi limitante ao crescimento das plantas. Esses autores atribuíram a tais resultados a concentração elevada de zinco encontrado na amêndoa $\left(56,12 \mathrm{mg} \cdot \mathrm{kg}^{-1}\right)$.

Corroborando com os resultados obtidos nesta pesquisa, que não demonstraram a necessidade de correção dos teores de $\mathrm{Zn}^{2+}$ e $\mathrm{Mn}^{2+}$, Mendonça et al. (1999), em estudos de casa de vegetação com mudas de aroeira (Myracrodruon urundeuva) observaram que as plantas cultivadas em um Cambissolo com teor de $0,7 \mathrm{mg} \cdot \mathrm{dm}^{-3} \mathrm{de} \mathrm{Zn}^{2+}$ e $14,4 \mathrm{mg} \cdot \mathrm{dm}^{-3} \mathrm{de} \mathrm{Mn}^{2+}$ não tiveram as características de crescimento afetadas pela omissão desses nutrientes, entretanto para o $\mathrm{Cu}^{+2}$ os resultados são divergentes, pois os autores não demonstraram a necessidade de sua aplicação.

Não houve alteração na produção de matéria seca da raiz em relação matéria seca da parte aérea, pois a relação raiz/parte aérea $(\mathrm{R} / \mathrm{PA})$ manteve-se equilibrada, não havendo diferença significativa entre os tratamentos (Tabela 1). As alteraçóes promovidas na parte aérea foram proporcionais às da raiz, não alterando essa relação. Esses resultados indicam que as plantas de mogno não desenvolveram o sistema radicular como mecanismo para compensar a deficiência de nutriente no solo. Segundo Clarkson (1985) é comum em ambiente de baixa fertilidade a relação R/PA ser maior e pode ser uma estratégia da planta para retirar o máximo de nutrientes.

O crescimento relativo das mudas de mogno pode ser observado na Tabela 2. Pelos resultados, observa-se que a matéria seca total das plantas sob omissão de $\mathrm{Cu}$ foram as mais afetadas, com redução de $83 \%$, seguidas do $\mathrm{Mn}$ com $22 \%$ e do Zn com $8 \%$. Sanches (1976) consideram deficiência severa de um nutriente quando a produção de matéria seca cai a $40 \%$ a menos do que o tratamento completo, fato esse observado para a omissão de $\mathrm{Cu}$. 


\section{CONCLUSÕES}

De acordo com os resultados obtidos neste trabalho e nas condições em que foi conclui-se que:

1 - O micronutriente limitante ao desenvolvimento das mudas de mogno foi o $\mathrm{Cu}^{2+}$ disponível quando o seu teor estiver igual ou abaixo de $0,08 \mathrm{mg} \cdot \mathrm{Kg}^{-1}$ de solo.

2 - A dose de $1,5 \mathrm{mg} \cdot \mathrm{Kg}^{-1}$ de $\mathrm{Cu}$ foi suficiente para corrigir a deficiência do nutriente, sendo portanto a recomendada, em uma primeira aproximação.

\section{BIBLIOGRAFIA CITADA}

Alvarez V.V.H.; Novais, R.F.; Barros, N.F; Reinaldo, C.B.; Lopes, A.S. Interpretação dos resultados das análises de solos. In: Ribeiro, A.C. et al. (Ed.). Recomendações para o uso de corretivos e fertilizantes em Minas Gerais. CFSEMG, Viçosa. p. 25-32.

Braga, J. M. 1983. Avaliação da fertilidade do solo: ensaios de campo. UFV, Viçosa. 102pp.

Braga, F.A.; Vale, F.R. do; Ventorin, N.; Aubert, E.; Lopes, G.A. 1995. Exigências nutricionais de quatro espécies florestais. Revista Árvore, 19(1): 18-31.

Barros, J.G. 2001. Adubação e calagem para formação de mudas de mogno (Swietenia macrophylla King). Dissertação de mestrado, Faculdade de Ciências Agrárias, Universidade Federal do Amazonas, Manaus, Amazonas. 63pp.

Clarkson, D.T. 1985. Adaptaçôes morfológicas e fisiológicas das plantas a ambientes de baixa fertilidade. In: Simpósio sobre reciclagem de nutrientes e agricultura de baixos insumos nos Trópicos. Anais. Ilhéus-BA: CEPLAC/SBCS, p.45-75.

Duboc, E.; Venturin, N.; Vale, F.R. Nutrição do jatobá (Himenaea courbaril L. Var. stilbocarpa (Hayene) Lee et lang). Cerne, 2(1): 138-152.

EMBRAPA. 1999. Manual de análises químicas de solos, plantas e fertilizantes. EMBRAPA, Brasília. 370pp.

Ferreira, D.F. Análises estatísticas por meio do Sisvar (Sistema para análise de Variância) para Windows 4.0. In: Reunião anual da região brasileira da Sociedade Internacional de Biometria, 45., São Carlos. Anais. São Carlos: UFSCar. p. 255-258.

Gomes, F.P. 1990. Estatística experimental. Piracicaba-SP: Nobel. 468pp.

Gullison, R. E.; Panfil, S.N.; Struose, J.J.; Hubbel, S.P. 1996. Ecology and management of mahogany (Swietenia macropylla King) in the Chimanes Forest, Beni, Bolivia. Botanical Journal of the Linnean society, 122: 9-34.

Lamprecht, H. 1990. Silvicultura nos Trópicos: ecossistemas florestais e respectivas espécies arbóreas - possibilidades e métodos de aproveitamento sustentado. Dt. Ges. fur. Techn. Zusammenarbeit (GTZ) GmbH, Eschoborn. (Trad. de Guilherme de AlmeidaSedas e Gilberto Calcagnotto). 343pp.
Lopes, A.S.; Carvalho, J.G. 1987. Métodos de diagnose da fertilidade do solo in: Fernandes, F. M.; Nascimento, V. M. (Coords). Curso de atualização em fertilidade do solo, Campinas: Fundação Cargil, 1987. p. 249-297.

Lorenzi, H. 1998. Árvores brasileiras: manual de identificação e cultivo de plantas arbóreas nativas do Brasil. Nova Odessa: Plantarum. 368pp.

Malavolta, E. 1980. Elementos de nutrição mineral de plantas. Agronômica Ceres, São Paulo. 251pp.

Malavolta, E.; Vitti, G.C.; Oliveira, S. A. 1989. Avaliação do estado nutricional das plantas: princípios e aplicaçôes. Associação Brasileira para Pesquisa da Potassa e do Fosfato. Piracicaba. 201pp.

Mendonça, A.V.R.; Nogueira, F.D.; Venturin, N.; Souza, J.S. Exigências nutricionais de Myracrodruon urundeuval (aroeira do sertão). Cerne, 5(2): 65-75.

Novais, R.F.; Neves, J.C.L.; Barros, N.F. 1991. Ensaio em ambiente controlado. In: Oliveira, A.J.; Garrido, W.E.; Araújo, J.D.; Lourenço, S. (Eds.). Métodos de Pesquisa em Fertilidade do Solo. Brasília: EMBRAPA-SEA, Brasília, Df. p. 189-255.

Pinto, F.P.da S.; Tucci, C.A.F.; Barros, J.G. Calagem e adubação na formação de mudas de mogno. In: IX Jornada de Iniciação Científica da Universidade do Amazonas. Manaus, AM. 2000. Resumos, p.5.

Raij, B.V. 1991. Fertilidade do solo e adubação. Agronômica. Ceres, São Paulo. 343pp.

Renó, N.B.; Siqueira, J.O.; Curi, N.; Vale, F.R. do. 1997. Limitações nutricionais ao crescimento inicial de quatro espécies arbóreas nativas em Latossolo Vermelho-Amarelo. Pesquisa Agropecuária Brasileira, 32(1): 17-25.

Sanches, P.A. 1981. Suelos de los trópicos: caracteristicas y manejo. San José: IICA. 660pp.

Sanches, P.A.; 1976. Propperties and management of soils in the tropics. New York, J. Willey. 618pp.

Sanginga, N.; Gwaje, D; Swift, M.J. 1991. Nutrient requeriments of exot tree species in Zinbabwe. Plant and Soil, The Hague, 132(2): 197-205.

Santos, R.A.C. 2006. Efeito de doses crescentes de nitrogênio, fósforo e potássio na produção de mudas de mogno (Swietenia macrophilla King) e pau-de-balsa (Ochroma pyramidale) e seleção de extratores de fósforo e potássio. Dissertação de Mestrado, Faculdade de Ciências Agrárias, Universidade Federal do Amazonas. Manaus, Amazonas. 63pp.

Silva, A.R.M. 2004. Calagem para formação de mudas de mogno (Swietenia macrophilla King) e sumaúma (Ceiba pentandra L. Gaertn). Dissertação de mestrado, Faculdade de Ciências Agrárias, Universidade Federal do Amazonas. Manaus, Amazonas. 63pp.

Simões, J.W.; Couto, H.T.Z. (1973). Efeitos da omissão na alimentação do pinheiro do Paraná (Araucária angustifólia) (Bert.) O. Ktze cultivado em vaso. IPEF. Piracicaba, 4(7): 3-40. 
Viegas, I.J.M.; Capucho, D.A.; Thomaz, M.A.A.; Conceição, H.E.O.; Pinheiro, E. 2004. Limitações nutricionais para o cultivo de açaizeiro em Latossolo Amarelo textura média, Estado do Pará. Revista Brasileira de Fruticultura, 26(2): 382-384.

Veríssimo, A; Barreto, P.; Tarifa, R; UHL, C. 1995. Extraction of a high-value natural resource in Amazônia: the case of mahogany. Forest Ecology and Management, 72(1): 39-60.

Recebido em 13/10/2006

Aceito em 23/05/2007 